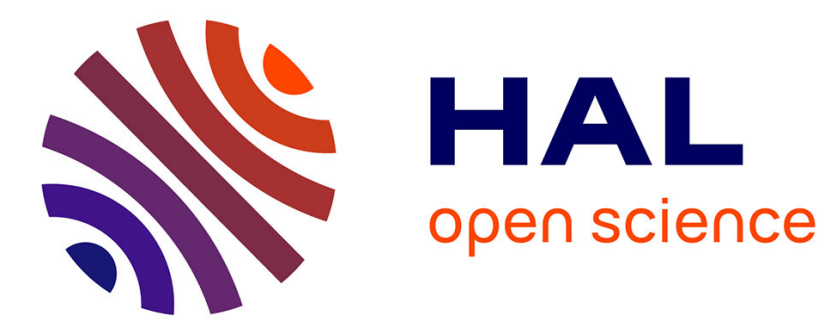

\title{
Static wetting behaviour of diblock copolymers
}

D. Ausserre, V. Raghunathan, M. Maaloum

\section{To cite this version:}

D. Ausserre, V. Raghunathan, M. Maaloum. Static wetting behaviour of diblock copolymers. Journal de Physique II, 1993, 3 (10), pp.1485-1496. 10.1051/jp2:1993215 . jpa-00247921

\section{HAL Id: jpa-00247921 https://hal.science/jpa-00247921}

Submitted on 1 Jan 1993

HAL is a multi-disciplinary open access archive for the deposit and dissemination of scientific research documents, whether they are published or not. The documents may come from teaching and research institutions in France or abroad, or from public or private research centers.
L'archive ouverte pluridisciplinaire HAL, est destinée au dépôt et à la diffusion de documents scientifiques de niveau recherche, publiés ou non, émanant des établissements d'enseignement et de recherche français ou étrangers, des laboratoires publics ou privés. 
Classification

Physics Abstracts

$68.10-68.15-61.30-61.25$

\title{
Static wetting behaviour of diblock copolymers
}

\author{
D. Ausserre, V. A. Raghunathan and M. Maaloum \\ Equipe de Physique de l'Etat Condensé. C.N.R.S. U.R.A. 807, Université du Maine. B.P. 535, \\ 72017 Le Mans Cedex, France
}

(Received 23 February 1993, revised and accepted 16 July 1993)

\begin{abstract}
Thin liquid films of ordered dihlock copolymers deposited on a solid substrate form a multilayer stacking parallel to the solid surface. A multilayer with a finite extend can he stable, metastable, or unstable, depending on the relative values of the surface energies of the various interfaces. The spreading parameter and chemical potential of a n-layer are derived, and used for classifying all possible situations. It is shown that only mono- and bilayers can be stable, and that non-wetting multilayers are subjected to a long-time piling up instability, leading in practice to the formation of characteristic ziggouratz-like Structures.
\end{abstract}

A diblock copolymer is made of two polymer chains with different chemical compositions $A$ and $B$ linked together end to end. Depending on the temperature, these materials are found in two different states. For $T>T_{\mathrm{OD}}, \mathrm{A}$ and $\mathrm{B}$ are mixed and form a homogeneous melt. On the other hand, for $T<T_{O D}, \mathbf{A}$ and $\mathrm{B}$ species phase-separate and form miccrodomains with one dimension at least comparable to the chain sire. $T_{O D}$ is called the microphase separation temperature or the order-disorder transition temperature [I]. In the case of symmetric diblock copolymers, which have almost equal volume fractions of $\mathrm{A}$ and $\mathrm{B}$, the microphase structure is lamellar. In thin films of these materials deposited on a solid substrate, these layers orient parallel to the substrate. In the present paper, we examine the wetting properties of these copolymer melts.

The wetting behaviour of a simple liquid $\mathbf{A}$ on a solid surface is controled by the spreading parameter [2], defined by

$$
S_{\mathrm{A}}=\gamma_{S V}-\gamma_{\mathrm{LS}}^{\mathrm{A}}-\gamma_{\mathrm{LV}}^{\mathrm{A}}
$$

where $\gamma_{S V}, \gamma_{L S}^{\mathrm{A}}$ and $\gamma_{L V}^{\mathrm{A}}$ are the surface tensions associated with the solid-vapour, liquid-solid and liquid-vapour interfaces, respectively. If $S_{\mathrm{A}}$ is positive, the liquid spreads on the solid surface. Otherwise it does not wet the surface and forms a drop with a well-defined contact angle $\theta_{\mathrm{A}}$ given by the Young relation

$$
\gamma_{L V}^{A} \cos \theta_{A}=\gamma_{S V}-\gamma_{L S}^{A}
$$

Equations (1) and (2) hold good for any homogeneous liquid including polymer melts 


\section{Spreading parameter of a monolayer.}

Consider a monolayer of a symmetric diblock copolymer lying on a solid substrate, as illustrated in figure 1. The free energy per chain in such a film can be written as [3]

$$
F_{c}=(312) \alpha k_{\mathrm{B}} T\left(\ell / R_{0}\right)^{2}+\left(N a^{3} / \ell\right)\left(\gamma_{\mathrm{AB}}+\gamma_{\mathrm{LV}}^{\mathrm{B}}+\gamma_{\mathrm{LS}}^{\mathrm{A}}\right)
$$

where $l$ is the monolayer thickness, a a numerical factor of the order of $1, k_{\mathrm{B}}$ the Boltzmann constant, $\mathrm{N}$ the monomer index of the polymer, $a$ the typical monomer size and $R_{0}=a N^{(1 / 2)}$. This phenomenological expression is found to be able to describe most of the observed properties of the lamellar phase of copolymers in the strong segregation limit, i.e. far from the order disorder transition. In the following we assume the above expression to be valid for a wide range of values of $\ell$.

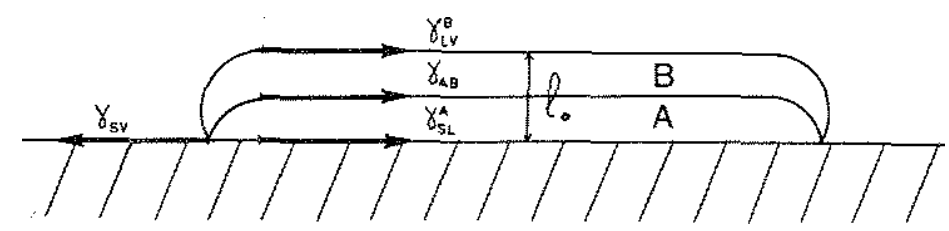

Fig. 1. - Schematic cross-section of a finite monolayer AB on a solid substrate. The equilibrium thickness $\ell_{0}$ is obtained for a balance of the different surface tensions, which can be viewed as forces per unit length of the edge.

The free energy of the system is therefore given by

$$
F_{1}=m F_{\mathrm{c}}+\gamma_{\mathrm{SV}}\left(A_{\mathrm{S}}-A_{\mathrm{P}}\right)
$$

where $A$, is the total surface area of the solid, $A$, the area covered by the copolymer and $m$ the total number of polymer chains in the melt. Taking the energy of the bare solid as the reference, one can replace $F_{1}$ by

$$
\Delta F_{1}=F_{1}-\gamma_{S V} A_{S} .
$$

Since $\mathrm{A},=m N a^{3} / \ell$, one gets

$$
\Delta F_{1}=m\left((3 / 2) \alpha k_{\mathrm{B}} T\left(\ell / R_{0}\right)^{2}-S_{1} N a^{3} / \ell\right)
$$

with

$$
S_{\mathrm{I}}=\gamma_{S V}-\gamma_{\mathrm{LS}}^{\mathrm{A}}-\gamma_{\mathrm{LV}}^{\mathrm{B}}-\gamma_{\mathrm{AB}}
$$

When $S_{1}$ is negative, $\boldsymbol{A} \boldsymbol{F}$, has a minimum at $\mathcal{\ell}=\ell_{0}$, with

$$
\frac{\ell_{0}}{R_{0}}=\left(-\frac{S_{1} a R_{0}}{3 \alpha k_{\mathrm{B}} T}\right)^{1 / 3}
$$

and

$$
\Delta F_{1}\left(\ell_{0}\right)=-(3 / 2) S_{1} \mathrm{~A}
$$


If one includes the possibility of multilayer stacking, the minimum of $\boldsymbol{A F}$, does not give necessarily the most stable state. Hence the rnonolayer at $\ell_{0}$ could he metastable. When $S_{\ell}>0, \Delta F$, has no minimum for any $\ell>0$ within the framework of equation ( 3 ), and the melt totally spreads on the solid surface. The final thickness of the film in this case will he determined by the disjoining pressure due to the van der Waals forces. The sign of $S_{1}$ therefore determines the wetting behaviour of the monolayer. Note that :

1) The spreading condition $S_{1}>0$ is much more difficult to realize than for a simple liquid, due to the additional surface tension $\gamma_{A B}$;

2) The fact that the two pure species $\mathbf{A}$ and $B$ taken separately wet a given solid surface, does not imply that the same is true for the copolymer;

3) We call $\mathbf{A}$ the species in contact with the solid, which implies :

$$
\gamma_{\mathrm{LS}}^{\mathrm{A}}+\gamma_{\mathrm{LV}}^{\mathrm{B}}<\gamma_{\mathrm{LS}}^{\mathrm{B}}+\gamma_{\mathrm{LV}}^{\mathrm{A}}
$$

\section{Energy of a copolymer bilayer.}

The structure of the bilayer requires that the same chemical species he in contact with both the solid and the vapour (Fig. 2). We take this to he the species A. In other words, we assume that the inequality,

$$
\gamma_{L S}^{\mathrm{A}}+\gamma_{L V}^{\mathrm{A}}<\gamma_{\mathrm{LS}}^{\mathrm{B}}+\gamma_{\mathrm{LY}}^{\mathrm{B}}
$$

is satisfied. In terms of $\Delta \gamma_{\mathrm{LS}}=\gamma_{\mathrm{LS}}^{\mathrm{B}}-\gamma_{\mathrm{LS}}^{\mathrm{A}}$ and $\Delta \gamma=\gamma_{\mathrm{LV}}^{\mathrm{B}}-\gamma_{\mathrm{LV}}^{\mathrm{A}}$, the above inequality can he written as

$$
A Y+\Delta \gamma_{L S}>0
$$

We will always assume in the following that both (8) and (9) are satisfied, which reads $\Delta \gamma_{\mathrm{LS}}>|\mathrm{Ay}|$. It means that we impose the preference of the solid for $\mathbf{A}$ to he stronger than the preference of the free surface for any species. If it was not the case, we should call 4 the polymer at the free surface and invert LV and LS indexes everywhere; then we could use directly all the results which follow. Indeed, as we consider geometries where the liquid-solid and liquid-vapor interface have the same area, they play a symmetric role in the expression of the free energy. Hence conditions ( 8 ) and (9) do not restrict the generality of the analysis.

Let $\ell_{1}$ and $\ell_{2}$ he the thicknesses of the two layers in the bilayer (Fig. 2). For fixed $A_{p}$, one can exchange molecules between the two Layers and hence $\ell_{1}$ and $\ell_{2}$ have to be determined by minimizing the energy of the system with respect to one of them. From volume conservation we have

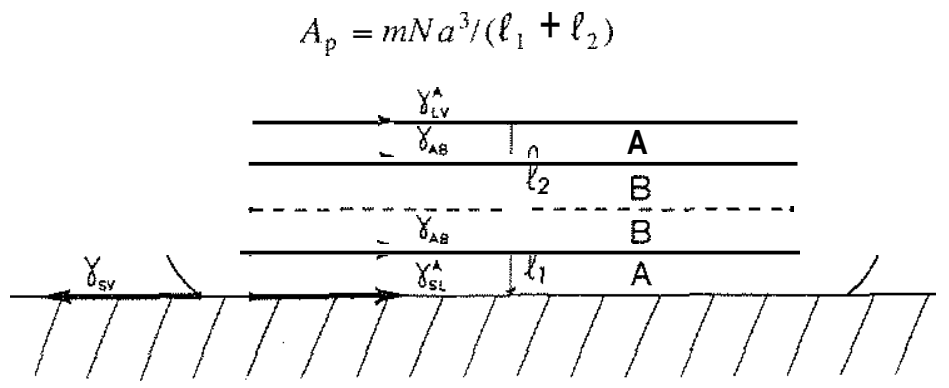

Fig. 2. - Schematic cross-section of a finite bilayer ABBA on a solid. The fictive interface BB is represented by dotted lines. 
The total energy of the system is

$$
\Delta F_{2}=(3 / 2) \alpha k_{\mathrm{B}} T\left\{m_{\mathrm{B}} \ell_{1}^{2}+m_{2} \ell_{2}^{2}\right\} / R_{0}^{2}+\left\{2 \gamma_{\mathrm{AB}}+\gamma_{\mathrm{I}}^{\mathrm{A}}+\gamma_{\mathrm{LS}}^{\mathrm{A}}-\gamma_{\mathrm{SV}}\right\} A_{\mathrm{P}}
$$

with $m_{1}=A, \ell_{1} /\left(\mathrm{Na}^{3}\right)$ and $m_{2}=A, \ell_{2} /\left(N a^{3}\right)$.

Minimization of $\Delta F_{2}$ with respect to $\ell_{1}$ at constant $A$, leads to $\ell_{1}=\ell_{2}$, i.e., the two layers have the same thickness. The minimum of the energy is given by

$$
\Delta F_{2}=m\left((3 / 2) \propto k_{B} T\left(\ell / R_{0}\right)^{2}-S_{2} N a^{3} / \ell\right)
$$

where

$$
S_{2}=(1 / 2) S_{\mathrm{A}}-\gamma_{\mathrm{AB}}
$$

Note that this is the same expression as that for AF, but with $S$, replaced by $S_{2}$. When $S_{2}<0, \mathrm{AF}_{2}$ has a minimum and the bilayer is at least a metastable state. For $S_{2}>0$, the bilayer is unstable and spreads on the solid surface.

3. Wetting behaviour of a multilager.

In the following discussion we assume that all the layers in the film have the same area. As we are considering only the stable and metastable states, this does not exclude any equilibrium possibility. For $n$ layers the expression for the free energy generalizes to

$$
\Delta F_{n}=m\left((3 / 2) \alpha k_{\mathrm{B}} T\left(\ell / R_{0}\right)^{2}-S_{n} N a^{3} / \ell\right) .
$$

All the layers in the film have the same thickness $\ell$, since they have the same area. The spreading parameter $\mathrm{S}$, now becomes

where

$$
S_{n}=(1 / n)\left(S_{\mathrm{A}}-\Delta \gamma^{\text {up }}\right)-\gamma_{\mathrm{AB}}
$$

$$
\begin{aligned}
\Delta \gamma^{\text {up }} & =y \text { for } n \text { odd } \\
& =0 \text { for } n \text { even }
\end{aligned}
$$

The multilayer is at least metastable if $\mathrm{S}_{,}<0$. This condition is always satisfied for sufficiently large 17 , since $S_{n} \rightarrow-\gamma_{\mathrm{AB}}$ as $n \rightarrow \mathrm{m}$. The free energy of a metastable $n$-layer state is

$$
\mathbf{A F},=-(3 / 2) n S_{n} A,
$$

with $A,=m N a^{3} /(n \ell)$. Minimizing equation (12) the equilibrium layer thickness is found to be

$$
\frac{\ell_{0}(n)}{R_{0}}=\left(-\frac{S_{n} a R_{0}}{3 \alpha k_{\mathrm{B}} T}\right)^{1 / 3}
$$

Dividing $O F$, by $m$ gives the chemical potential

$$
\mu_{n}=\left(\frac{3}{2}\right)\left(3 \alpha k_{\mathrm{B}} T\right)^{1 / 3}\left(a R_{0} S_{n}\right)^{2 / 3}
$$

As $\mu_{n}$ is a monotonic function of $\left|S_{n}\right|$, all the stability analysis can be easily carried out in terms of the spreading parameter.

Dividing $\mathrm{S}$, by $\gamma_{\mathrm{AB}}$, one gets the dimensionless spreading parameter

$$
\sigma_{n}=(1 / n)\left(\sigma_{\mathrm{A}}-\delta^{\mu \mathrm{P}}\right)-1
$$


where

$$
\sigma_{\mathrm{A}}=S_{\mathrm{A}} / \gamma_{\mathrm{AB}} \quad \text { and } \quad \delta^{\mathrm{up}}=\Delta \gamma^{\mathrm{up} / \gamma_{\mathrm{AB}}}
$$

Note that

$$
\begin{array}{rlr}
\delta^{\text {up }} & =\delta=\left(\gamma_{\mathrm{LV}}^{\mathrm{B}}-\gamma_{\mathrm{LV}}^{\mathrm{A}}\right) / \gamma_{\mathrm{AB}} & \text { for } n \text { odd } \\
& =0 & \text { for } n \text { even }
\end{array}
$$

These dimensionless quantities are used in the following discussion. As the behaviour of the system is determined by the sign of $\mathrm{d} \sigma_{n} / \mathrm{d} n$ and the sign and magnitude of $\sigma_{n}$, we consider the various possibilities below.

$3.1 \sigma_{\mathrm{A}}-\delta^{\text {up }}<0$. - In this case $\mathrm{U}_{\text {, }}$ is negative for all $n$ all states are metastable. Further, as $\mu_{n}$ decreases with increasing $n$, a given film would tend to decrease its surface area by increasing the number of layers in it. This results in what may be called a piling up instability. Figure 3 illustrates the situation where condition 3.I holds for both parities. Note that the continuous lines in the figure are only guides for the eye, since $\sigma_{n}$ is defined only for integer values of $n$. Figure 4 shows an experimentally observed example of such a piled-up ziggouratlike structure obtained after very long time from an initially uniform 3-layers film. Because of its circular symmetry, we call it Babel tower. Each layer in this pyramidal structure has a different area, suggesting that it is a transient state involved in the evolution process.

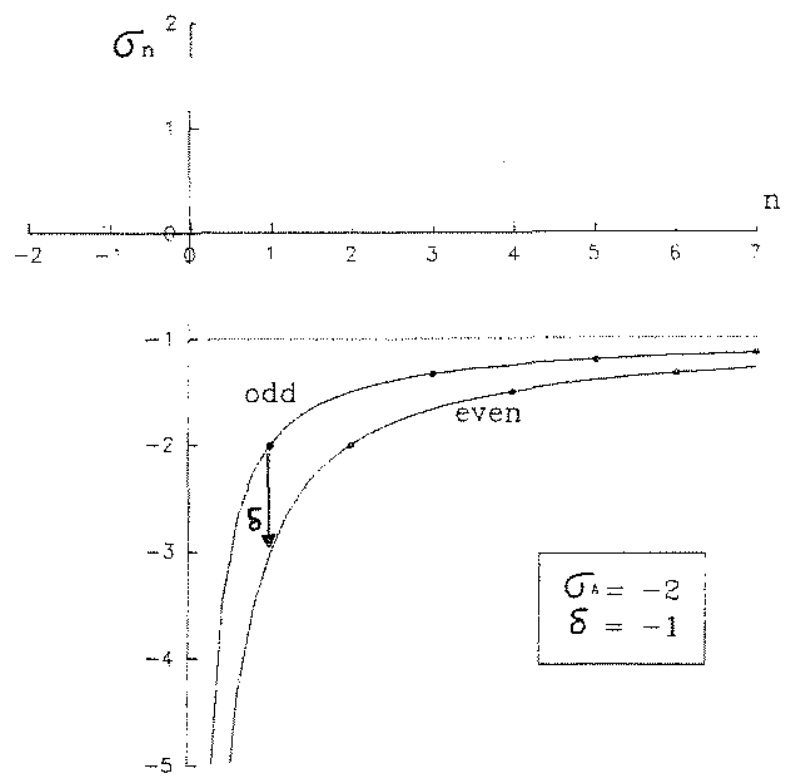

Fig. 3. - Variation of $\sigma_{n}$ versus $n$ for $\sigma_{\mathrm{A}}=-2$ and $\delta=-1 . \sigma_{n}$ is only defined for integer values of $n$, corresponding to big dots on the two continuous curves. The chemical potential $\mu_{s}$ is monotonic function of the distance between the $n^{\text {th }}$ dot and the horizontal axis. A preference far a high number of layers is therefore obtained in that example. 


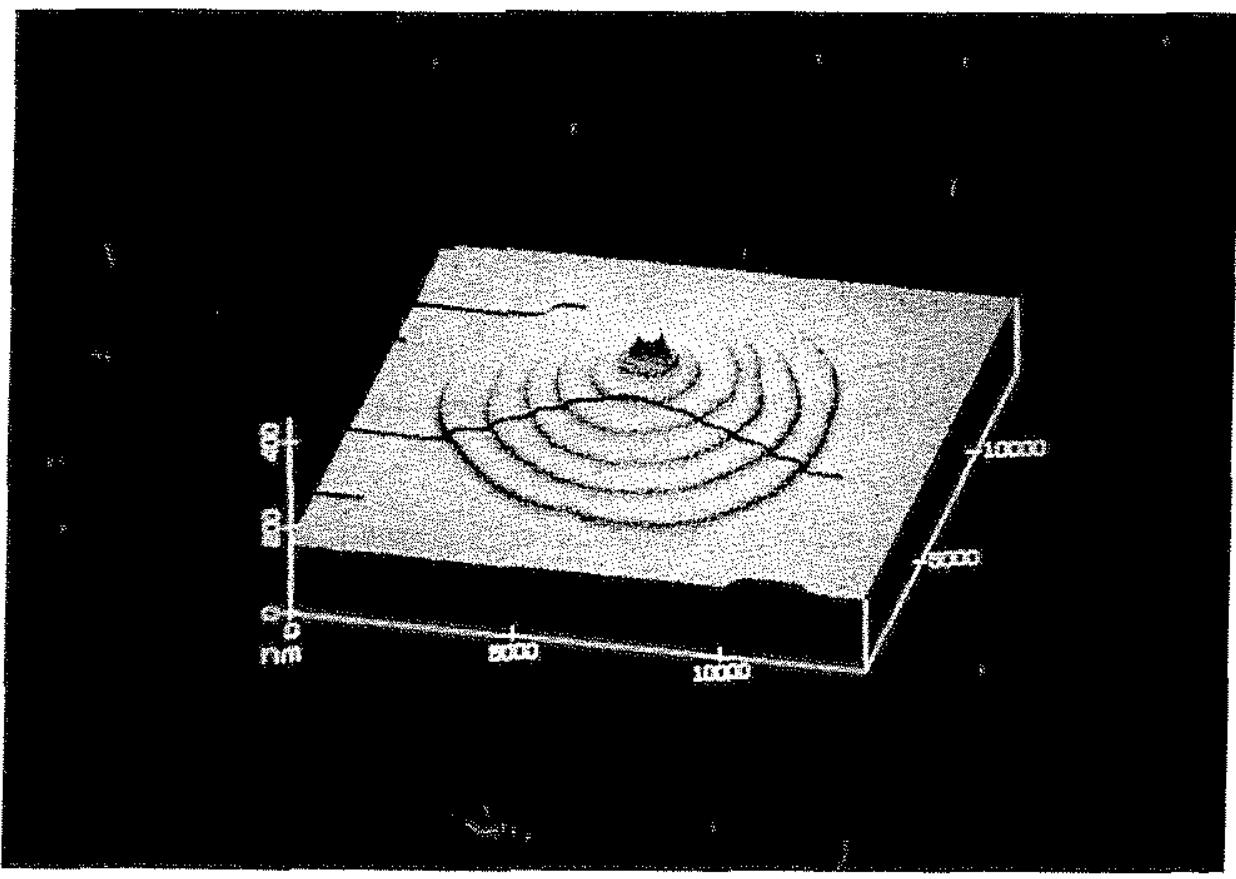

Fig. 4. - Ziggourat-like structure obtained after annealing at $170^{\circ} \mathrm{C}$ for one day a thin film of a symmetric (Polystyrene-polymethyimetacrylate) diblock copolymer of mass 57000 , with a thickness initially uniform, as observed by Atomic Force Microscopy. Each step between two circular terraces has the same height $\boldsymbol{L}=290 \pm 10 \AA$, identical to the lamellar periodicity.

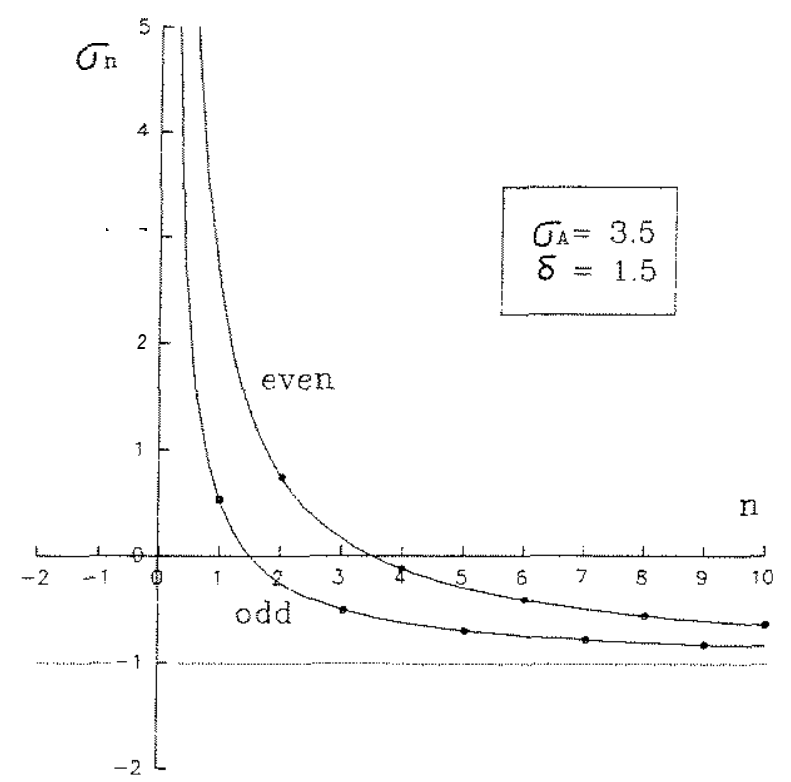

Fig. 5. $-\sigma_{n}$ versus $n$ for $\sigma_{\mathrm{A}}=5.5$ and $\delta=2$. $n$-layers corresponding to big dots above the $n$-axis are spreading completely on the solid substrate. Their chemical potential is not defined. Dots below the naxis correspond to metastable state, with one preferential state $n$ * for each parity. In this example, the lowest chemical potential is obtained for $n^{*}$ even, which is only metastable however. 
3.2 $\sigma_{\mathrm{A}}-\delta^{\text {山P }} \gg 0$. - In this section we consider situations where $\sigma_{A}-\delta$ is high enough so that $\sigma_{n}>0$ for at least one value of $n$. In other words, $\sigma_{A}>2$ if $n$ is even and $\sigma_{\mathrm{A}}>1+\delta$ if $n$ is odd. On increasing $n, u$, becomes negalive at $n=n^{*}$ and tends to $-\gamma_{\mathrm{AB}}$ as $n \rightarrow \infty$. Thus for $17<n^{*}$, the condition for spreading is satisfied. For $n \geqslant n^{*}$, metastable states are obtained with the state $n=n^{*}$ having the lowest energy. Note that even this state is only metastable as the energy of the film decreases without bound when it spreads on the solid surface. This situation is illustrated in figure 5.

\section{$\mathbf{3 . 3}$ INTERMEDIATE CASE.}

$$
\begin{array}{ll}
\delta<\sigma_{\mathrm{A}}<1+\delta & (n \text { odd }) \\
0<\sigma_{\mathrm{A}}<2 &
\end{array}
$$

3.3.1. - In the narrow range of values of $\sigma_{\mathrm{A}}$ defined by the above two conditions, $\sigma_{n}$ is again a decreasing function of $n$, but it is always negative, Therefore, a given film cannot spread. All states are metastable and the chemical potential increases with $n$. Hence there is one absolutely stable state which is either the monolayer or the bilayer. It is a bilayer if $\mu_{2}<\mu_{\text {f }}$, i.e., if

$$
\sigma_{\mathrm{A}}<2 \delta
$$

Figure 6 shows the variation of $\sigma_{n}$ with $n$ for such a situation. A necessary condition for satisfying the above three conditions is that $0<\delta<2$.

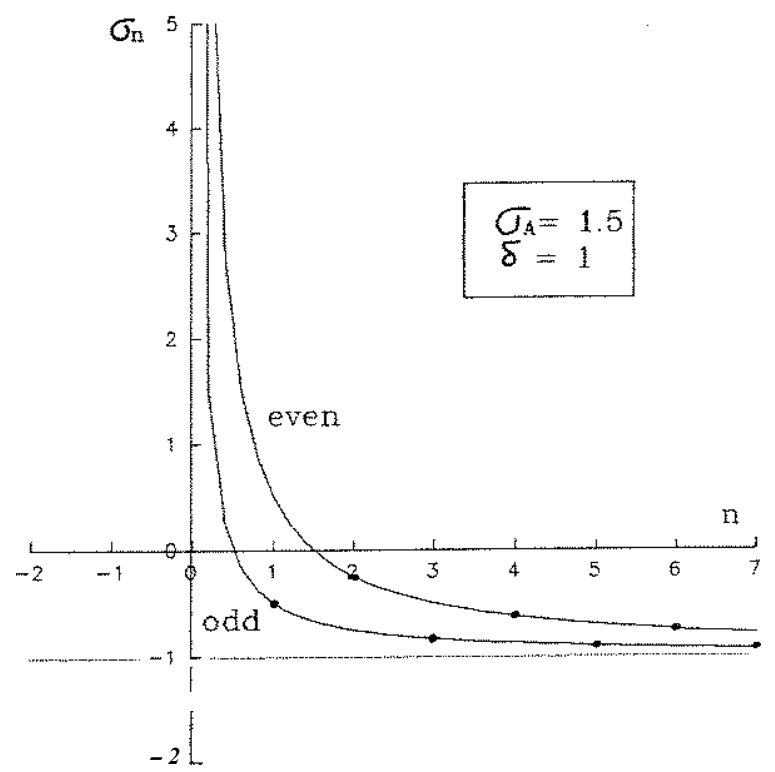

Fig. 6. $-\sigma_{n}$ versus $n$ for $\sigma_{\mathrm{A}}=1.5$ and $\delta=1$. There is no spreading n-layer since no dot is found above the n-axis. The big dot which is the closest from the axis is at $n=2$. The bilayer is therefore stable.

3.3.2. - Let us now consider a situation where (17) is satisfied but not (18). There are two possibilities; either $\sigma_{\mathrm{A}}<0$ or $\sigma_{\mathrm{A}}>2$. 
i) $\sigma_{\mathrm{A}}<0$.

A necessary condition is that $\delta<0$. Then the even $n$ states fall into case a and are all metastable with the chemical potential decreasing with increasing $n$. The chemical potential of any odd state is smaller than that of any of the even parity states. If the system is initially in an even state, it can be expected to switch parity and finally reach the absolutely stable monolayer. This situation is illustrated in figure 7 .

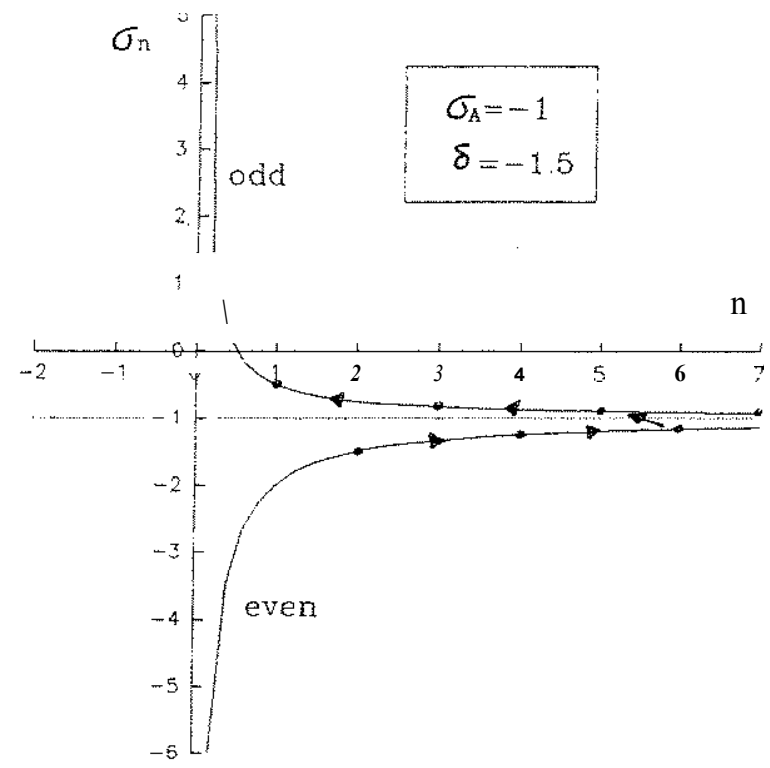

Fig. 7. -0 , yersus $n$ for $\sigma_{\mathrm{A}}=-1$ and $\delta=-1.5$. The monolayer is absolutely stable. A possible evolution of an initial bilayer is shown by the arrows.

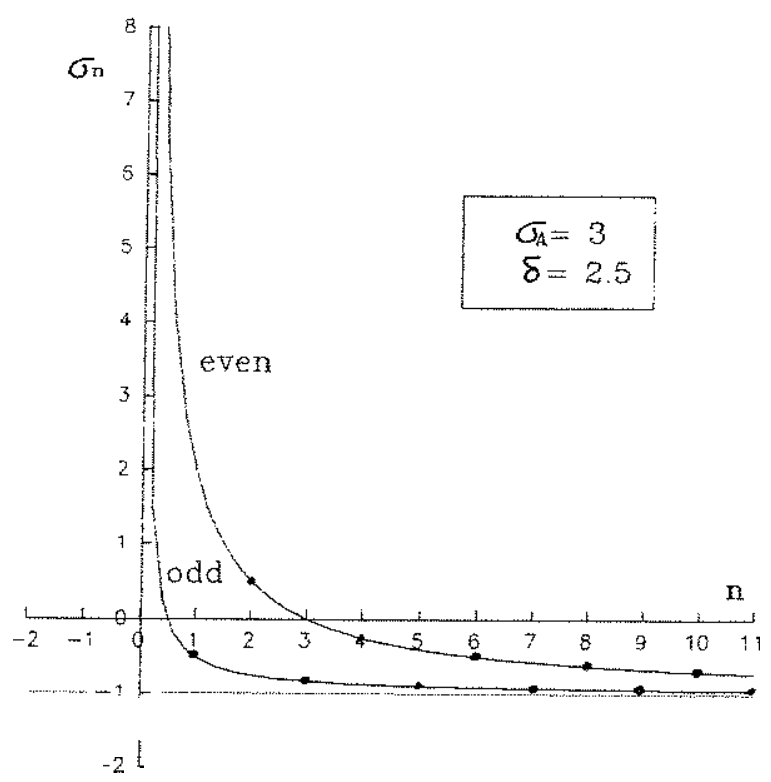

Fig. 8. $-\sigma_{n}$ versus $n$ for $\sigma_{\mathrm{A}}=3$ and $\delta=2.5$. The monolayer is only metastable, since the dot for the bilayer is above the $n$-axis, meaning that the bilayer is spreading. 
ii) $\sigma_{A}>2$.

A necessary condition is that $\mathrm{S}>1$. In this case the bilayer and possibly some of the higher order even slates are unstable and spread on the solid surface. The monolayer is metastable as switching to the bilayer leads to complete wetting, thus lowering the energy of the system. Such a case is illustrated in figure 8 .

3.3.3. - Wefinally consider the case where (18) is satisfied but not (17). There are again two possibilities; either $\sigma_{\mathrm{A}}<\delta$ or $\sigma_{\mathrm{A}}>1+\delta$.

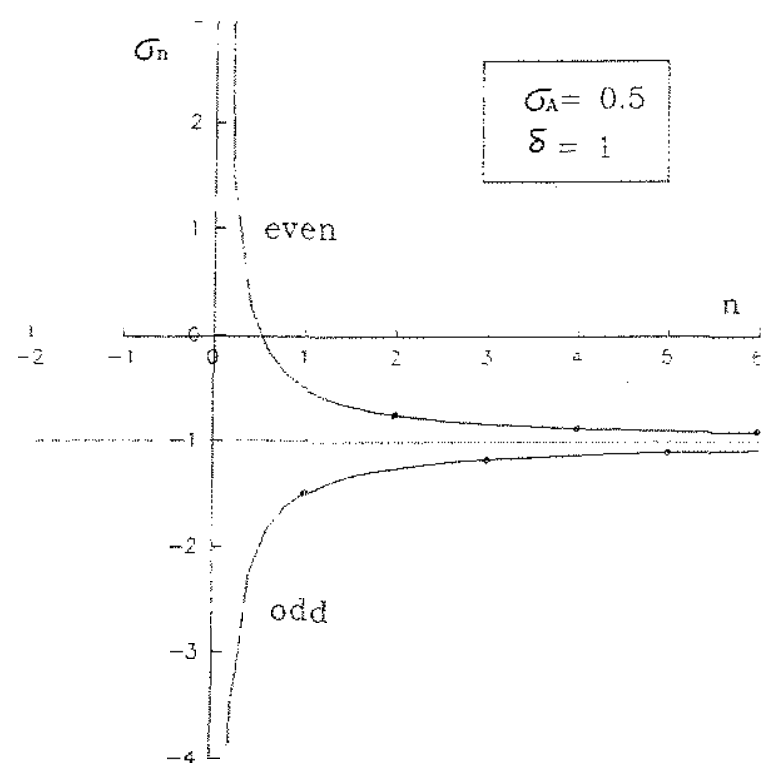

Fig. 9. $-\sigma_{4 t}$ versus $n$ for $\sigma_{\mathrm{A}}=0.5$ and $\delta=\mathrm{I}$. Odd states are piling up, while even states prefer lowest n. The bilayer is absolutely stable.

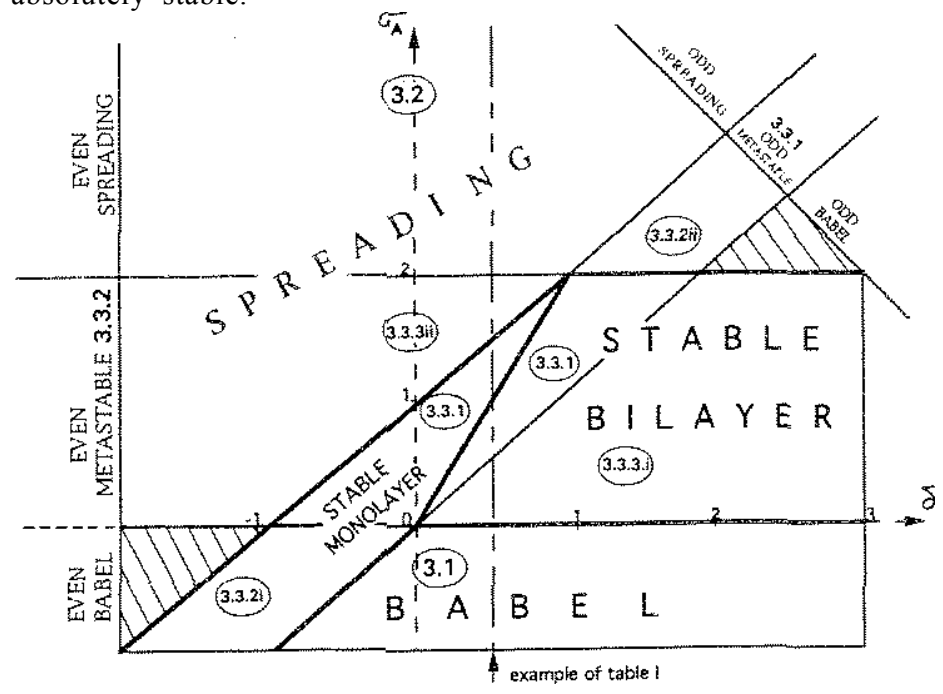

Fig. 10. - Dlagramm of ail possible regimes in the $\left(8, \sigma_{\mathrm{A}}\right)$ space. Types enclosed in ellipses refer to :he corresponding sections in the text. Heavy lines define four main regimes defined on the basis of the lowest energy state : spreading; monolayer; bilayer; and Babel. Thin lines divide these principal areas in subregions with uniform behaviours. The pathologic situations mentioned in the text are found in dashed arras. 
i) $\sigma_{A}<\delta$.

The necessary condition on $\mathrm{S}$ is that $\delta>0$. This situation is similar to the one of figure 7 (section $3.3 .2 ;$ i)) with odd and even parities inverted. The bilayer is now absolutely stable as can be seen from figure 9 .

iij $\sigma_{\mathrm{A}}>1+\delta$.

A necessary condition on $\mathrm{S}$ is that $\delta<1$. The odd parity states fall into case 3.2 and spread. The bilayer is now metastable. The case is very similar to the one illustrated in figure 8 for opposite parities.

All the discussion above has been summarized in the diagramm of figure 10 drawn in the $\left(\delta, \sigma_{\mathrm{A}}\right)$ space. To illustrate how to use it in practice, let us consider an example with $\delta=112$. Depending on $\sigma_{A}$, several behaviours can he encountered. They are graphically summarized below :

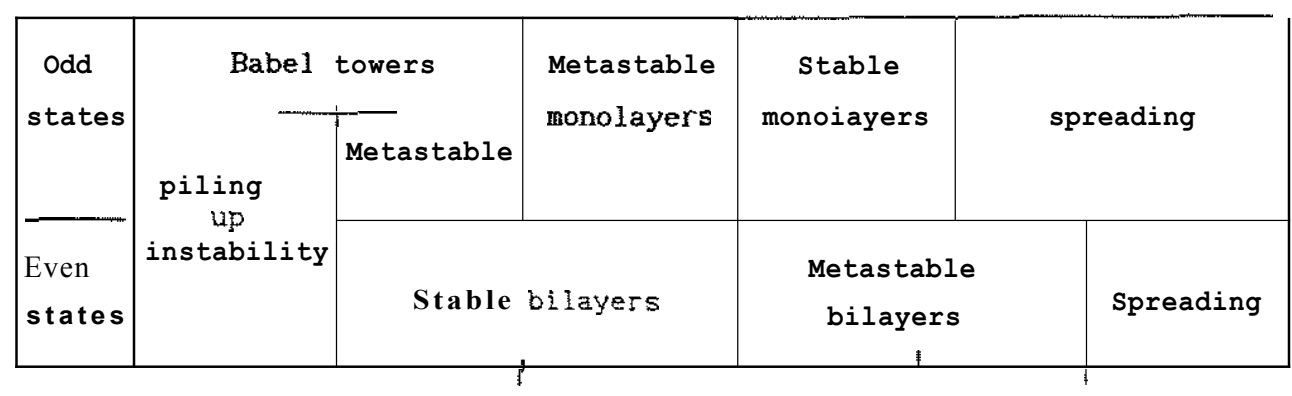

The two dashed area have been left apart in the discussion. They correspond to the strange situation where films belonging to one parity are spreading while those belonging to the other are piling up.

\section{Grafted monolayer.}

So far in this paper we have assumed that the polymer chains are free to move along the solid surface. However, in many practical situations it need not he so. Therefore, we now consider the case where the first copolymer monolayer is grafted onto the solid substrate. Then the solid surface behaves like a plane polymer melt surface. For connection with the previous sections, one assumes that the species B is grafted and that the species $\mathbf{A}$ is exposed to the air. One should also relax the earlier conventions embodied in (8) and (9) concerning A and B denominations. Further, the quantities $\gamma_{S \psi}, \gamma_{\mathrm{LV}}^{\mathrm{A}}, \gamma_{\mathrm{LS}}^{\mathrm{B}}$ have to be replaced respectively by $\gamma_{\mathrm{LV}}^{\mathrm{A}}, 0, \gamma_{\mathrm{AB}}, 0$ and $\Delta \gamma-\gamma_{\mathrm{AB}}$.

- If we have $\mathbf{A}$ against the surface, the dimensionless spreading parameter is given by

$$
\sigma_{\mu t}=-(1 / n) \delta^{\text {up }}-1
$$

If $\mathrm{S}$ is positive even states are prefered. They are all stable with the same chemical potential. Consequently, rough surfaces can develop with time. On the other hand, if $\delta<0$, odd states are prefered, with : i) stable monolayer and metastable multilayers if $-1<\delta<0$; ii) all states spreading if $\delta<-1$. 
- If we have $B$ against the surface, then

$$
\begin{array}{ll}
\sigma_{n}=-(1 / n)-1 & n \text { odd } \\
\sigma_{n}=-(1 / n)(1+\delta)-1 & n \text { even }
\end{array}
$$

A necessary condition for the surface to prefer $B$ over $\mathbf{A}$ is that

$$
\left|\sigma_{n}\right| \leqslant 1
$$

This is possible for the even states if $\left(d \sigma_{n} / \mathrm{d} n\right)<0$, i.e., if $\delta<-1$. But this is precisely the spreading condition when $\mathbf{A}$ is against the surface. Hence no stable state can be obtained with B against the surface.

As an additional remark we underline that moving $\delta$ across 0 corresponds in principle to a rougbening transition. Since $\gamma_{\mathrm{AB}}, \gamma_{\mathrm{LV}}^{\mathrm{B}}$ and $\gamma_{\mathrm{LV}}^{\mathrm{A}}$ are temperature dependent, it should be possible to obtain a temperature controled roughening transition by the right choice of $\mathbf{A}$ and $\mathbf{B}$. As an example, let us mention the polystyrene/polymethylmethacrylate copolymer as a reasonable candidate.

\section{On a possible reversible temperature induced wetting-dewetting transition.}

Consider a stable monolayer of thickness $\ell_{0}$ lying on a solid substrate. On increasing the temperature above $T_{O D}$, the film melts into a homogeneous mixture of the two species $\mathbf{A}$ and $\mathbf{B}$. As the A-B interface has disappeared, the spreading condition is now that for a simple liquid given by equation (1). It is possible that $S_{M}>0$ for the homogeneous melt, although $S_{1}<0$ for the monolayer, as the former expression does not contain the $-\gamma_{\mathrm{AB}}$ term. Hence we can induce a reversible wetting-dewetting transition by changing the temperature. The

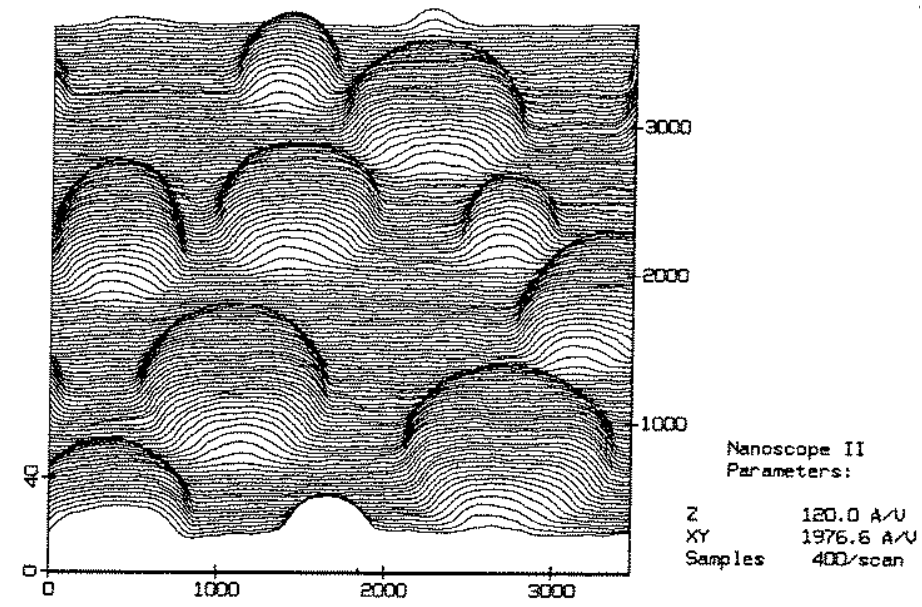

Data taken Fri Der 18 15:49:03 1992

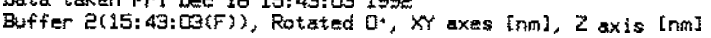

Fig. 11. - Disconnected islands of height $\ell_{0}(1)=160 \pm 10 \AA$ visualized by atomic force microscopy. The copolymer is a symmetric PS-PMMA dibloc of molecular mass 57000 , and the substrate a silicon wafer. The initial film was solid and homogeneous in thickness. The present structure has been obtained by annealing it at $T<T_{\text {OD }}$ for 24 hours. Further annealing for one month was unable to modify the island population. 
topology of the monolayer obtained on cooling an initially spread film of thickness $\ell$ at $T>T_{\text {OD }}$ can be expected to depend on $\ell$. If $\ell \ll \ell_{0} / 2, \ell_{0}$ being the equilibrium monolayer thickness, the monolayer will consist of disconnected islands of thickness $\ell_{0}$. Once formed, no kinetic evolution is possible except by diffusion of the islands as a whole. Such a structure is shown in figure II. On the other hand, if $\ell_{0} / 2 \ll \ell<\ell_{0}$, the monolayer would have a distribution of holes in it. A slow growth process driven by the line tension should lead to their ultimate disappearance. If $\ell \sim \ell_{0} / 2$, the dewetting should result in the formation of a hicontinuous labyrinth of wet and dewet regions. The slow evolution process can then he expected to lead to several large disconnected domains.

To summarize, the essential conclusion of the present work is that the possible equilibrium states of a non-grafted copolymer film reduce to monolayers or bilayers. Every n-layer with $n>2$ is therefore unstable (spreading) or metastable (piling up). The second important feature is that the ordered copolymer is more likely to he non-wetting compared to the homogeneous melt of the same composition. This leads to the possibility of a temperature controled wettingdewetting transition, driven by the appearance or disappearance of the $\mathbf{A}-€ \mathbf{3}$ interface. The third feature is the formation of characteristic Babel towers in the piling up instability. Their shape, however, cannot he understood within the static picture used in this study. The case of a grafted monolayer leads to the interesting possibility of the spontaneous roughening of the copolymer film deposited on it. A complete understanding of all the experimentally observed behaviours would now require to go into the dynamics of these systems.

\section{References}

[1] Bates F. S., Fredrickson G. H., Annu. Rev. Phys. Chem. 41 (1990) 525.

[2] de Gennes P. G., Rev. Mod. Phys. 57 (1985) 827.

[3] See e.g. Turner M. S., Phys. Rev. Lett. 69 (1992) 1788. 